\title{
An unusual case of an ulcerative colitis flare resulting in disseminated intravascular coagulopathy and a bladder hematoma:
}

\section{a case report}

\author{
David L Suskind*, Karen Murray and Dennis Christie
}

\author{
Address: Department of Pediatrics, Seattle Children's Hospital and Regional Medical Center, University of Washington, 4800 Sand Point Way NE, \\ Seattle, Washington, USA \\ Email: David L Suskind* - david.suskind@seattlechildrens.org; Karen Murray - karen.murray@seattlechildrens.org; \\ Dennis Christie - dennis.christie@seattlechildrens.org \\ * Corresponding author
}

Published: 07 October 2004

BMC Gastroenterology 2004, 4:26 doi:10.1 I86/147I-230X-4-26

This article is available from: http://www.biomedcentral.com/I47I-230X/4/26

(C) 2004 Suskind et al; licensee BioMed Central Ltd.

This is an open-access article distributed under the terms of the Creative Commons Attribution License (http://creativecommons.org/licenses/by/2.0), which permits unrestricted use, distribution, and reproduction in any medium, provided the original work is properly cited.

\begin{abstract}
Background: Disorders of coagulation have long been associated with inflammatory bowel disease. Children, as well as adults, with both active and inactive ulcerative colitis have been found to have abnormal coagulation and fibrinolysis. Disseminated intravascular coagulation arises from an overwhelming of the haemostatic regulatory mechanisms leading to an excessive generation of thrombin and a failure of the normal inhibitory pathways to prevent systemic effects of this enzyme. Ulcerative colitis has been associated with disseminated intravascular coagulation in conjunction with septicemia, toxic megacolon and surgery.
\end{abstract}

Case presentation: A fourteen-year-old boy with a history of poorly controlled ulcerative colitis presented with nonbilious emesis, hematochezia, and hematuria. Laboratory workup revealed disseminated intravascular coagulation. He was placed on triple antibiotics therapy. An infectious workup came back negative. A computerized tomography (CT) scan of the abdomen revealed a marked thickening and irregularity of the bladder wall as well as wall thickening of the rectosigmoid, ascending, transverse, and descending colon. Patient's clinical status remained stable despite a worsening of laboratory values associated with disseminated intravascular coagulation. Patient was begun on high dose intravenous steroids with improvement of the disseminated intravascular coagulation laboratory values within 12 hours and resolution of disseminated intravascular coagulopathy within 4 days. A thorough infectious workup revealed no other causes to his disseminated intravascular coagulation.

Conclusions: The spectrum of hypercoagulable states associated with ulcerative colitis varies from mild to severe. Although disseminated intravascular coagulation associated with ulcerative colitis is usually related to septicemia, toxic megacolon or surgery, we present a case of an ulcerative colitis flare resulting in disseminated intravascular coagulation and a bladder hematoma. 


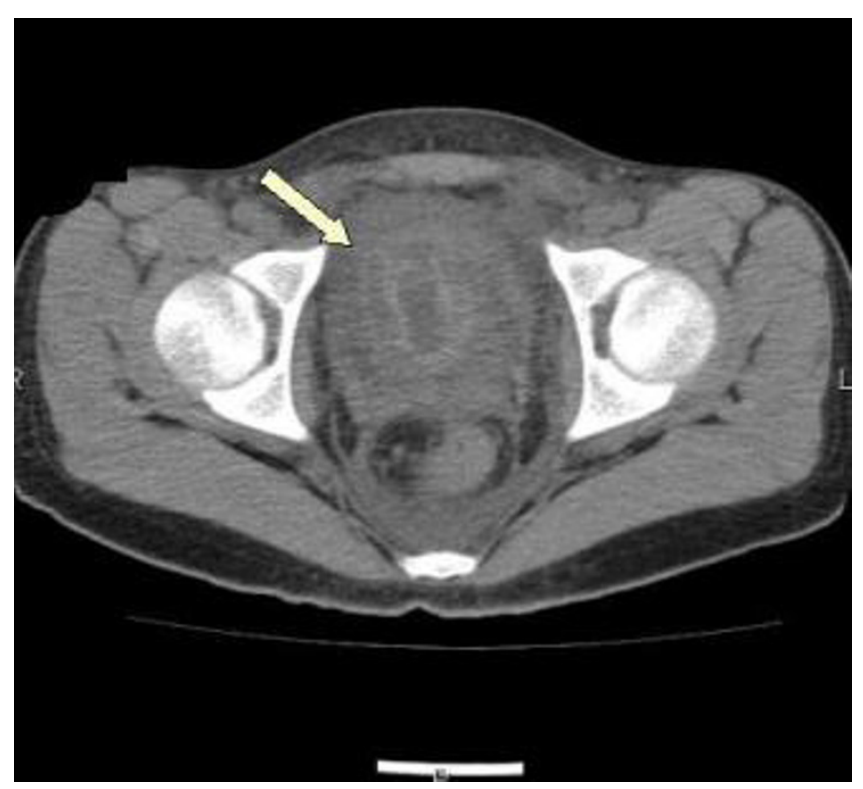

Figure I

Abdominal CT revealing a marked thickening and irregularity of the bladder wall consistent with bladder hematoma.

\section{Background}

A wide variety of disorders are associated with the development of disseminated intravascular coagulation (DIC). Initiation usually involves mechanical tissue injury and or endothelial cell activation and injury. DIC arises from an overwhelming of the haemostatic regulatory mechanisms leading to an excessive generation of thrombin and a failure of the normal inhibitory pathways to prevent systemic effects of the enzyme leading to DIC [1]. Ulcerative colitis has been associated with DIC. In previously reported cases, DIC has arisen from active disease in conjunction with septicemia, toxic megacolon or surgery [2-5]. The authors report a pediatric case of DIC associated with a colitis flare resulting in a bladder hematoma.

\section{Case presentation}

A 14-year-old boy with a diagnosis of ulcerative colitis based on colonic histology, serology and a normal barium study of his small bowels was admitted with a five-day history of nonbilious vomiting and bloody diarrhea. Additional symptoms included recent onset hematuria, and low-grade fevers to $100.4 \mathrm{C}$ over the prior four days. He had also sustained a $25 \mathrm{lb}$ weight loss in the last six months, indicating a lack of disease control.

As an outpatient, his maintenance therapy included mesalamine ( 1 gram three times a day), and mercaptopurine (75 mg once per day). In addition, he had been started on prednisone approximately 7 weeks prior for treatment of an ulcerative colitis flare. His current dose of prednisone was $10 \mathrm{mg}$ once a day. Soon after symptoms begun, he had been placed on ciprofloxacin as treatment for a presumptive flare.

Physical exam showed he was afebrile, with a heart rate of 130 beats per minute, respiratory 16 breaths per minute and blood pressure 115/67 mmHg. He was alert although with a sallow appearance. Abdominal exam revealed a soft nontender nondistended abdomen. Rectal showed normal external exam with grossly bloody stool. Initial blood work showed hemoglobin of 12.3, a normal white blood cell count, normal differential and normal platelet count with a mildly elevated prothrombin time of 16.2 with an international normalized ratio (INR) of 1.2. Urine analysis showed a specific gravity of 1.035, 3+blood, +ketones and $>100$ RBC per high powered field and 0-5 WBC per high power field. Abdominal ultrasound revealed irregular shaped bladder wall.

Patient was placed on intravenous fluids (IV) as well as metronidazole (IV). Blood and urine cultures were sent for analysis. Stool was sent for culture and for Clostridium difficile toxin analysis.

Serial repeat lab works the following day revealed a dropping hemoglobin $(7.4 \mathrm{~g} / \mathrm{dL})$ and platelet count $(64 \mathrm{~K} /$ $\mathrm{mm}^{3}$ ) increasing PT/PTT (21.3/47 seconds) with an INR of 1.8. Blood smear showed moderate amount of elliptocytes, schistocytes, microcytes and fragmented red blood cells. Initial DIC panel revealed an elevated D-dimer of $4.9 \mathrm{mcg} / \mathrm{mL}$ with a normal thrombin time and fibrinogen. Thrombin time subsequently increased to $>120$ seconds. D-dimers increased to $10.3 \mathrm{mcg} / \mathrm{mL}$. A computerized tomography (CT) scan of the abdomen revealed a marked thickening and irregularity of the bladder wall as well as wall thickening of the rectosigmoid, ascending, transverse, and descending colon (Figure 1). Urology was consulted and felt that this represented a submucosal hematoma.

Patient was begun on broad-spectrum antibiotics because of concerns regarding possible bacteremia and a worsening DIC laboratory picture. Blood, stool and urine cultures returned negative. Viral cultures and monoclonal antibody staining for adenovirus detection in the urine was negative. Despite a worsening in the DIC panel, the patient remained clinically unchanged. IV steroids were begun approximately 36 hours into patient's hospital stay. Patient had a stabilization of PT/PTT/INR/thrombin time and D-dimer, and a subsequent normalization of labs over the following 4-day period ( Figure 2, 3, 4, 5, 6, 7, 8 ). Patient's diarrhea and hematuria resolved as well. Colonscopy revealed chronic colitis consistent with ulcerative colitis. Cystoscopy revealed a fibrin clot consistent 


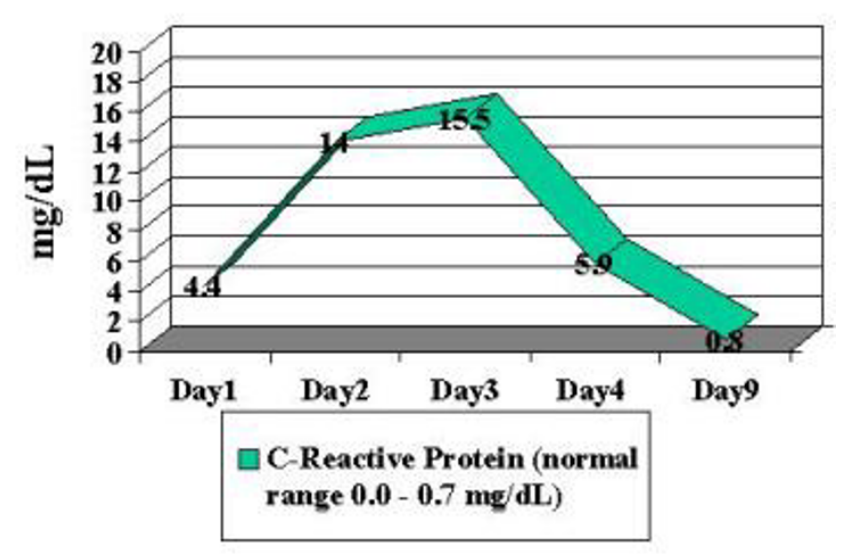

Figure 2

Graphic illustration of C-reactive protein throughout hospitalization: Day I (admission date) - Day 9 (day of discharge). Patient received intravenous steroids at approximately 36 hours into hospitalization.

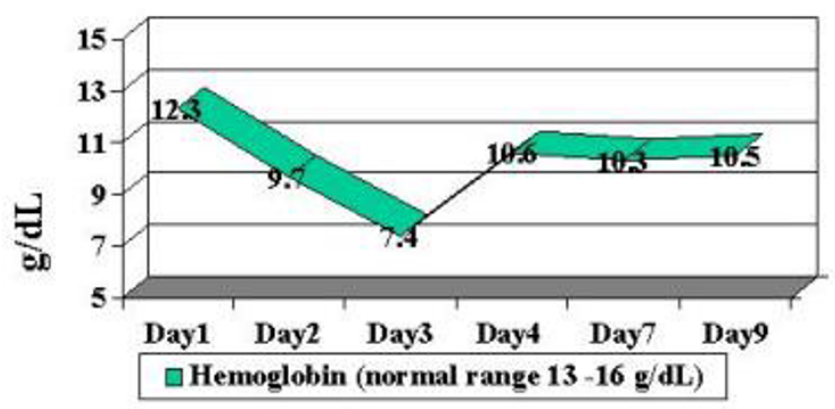

Figure 3

Graphic illustration of hemoglobin throughout hospitalization: Day I (admission date) - Day 9 (day of discharge). Patient received intravenous steroids at approximately 36 hours into hospitalization.

with submucosal hematoma. Patient was discharged from the hospital on a steroid taper, and remains in remission to date.

\section{Conclusions}

Disorders of coagulation have long been associated with inflammatory bowel disease [6-11]. Children, as well as adults, with both active and inactive ulcerative colitis have been found to have abnormal coagulation and fibrinolysis[11]. It is unclear whether this is a direct or indirect result of inflammatory bowel disease.

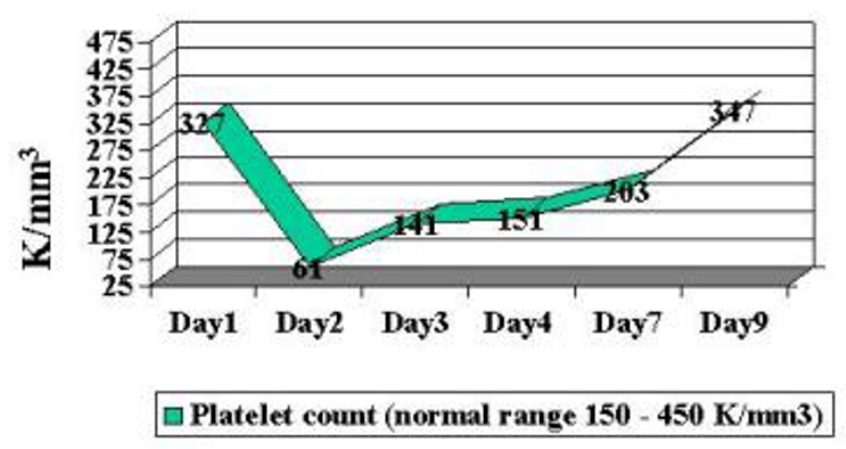

Figure 4

Graphic illustration of platelets throughout hospitalization: Day I (admission date) - Day 9 (day of discharge). Patient received intravenous steroids at approximately 36 hours into hospitalization.

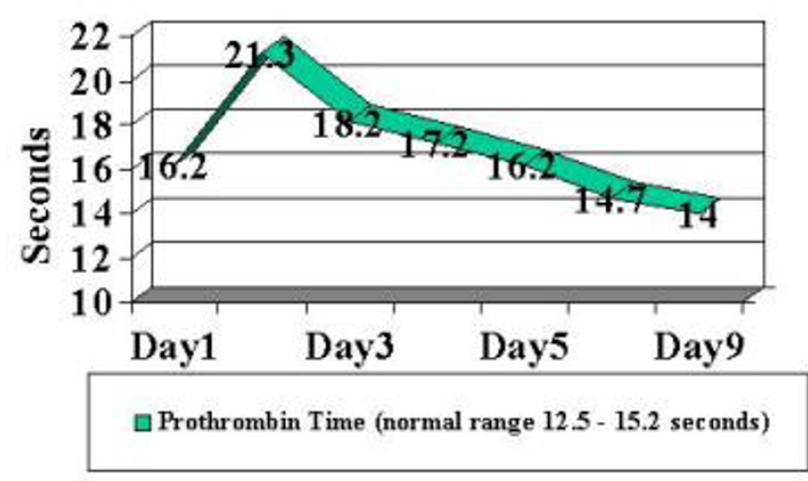

Figure 5

Graphic illustration of prothrombin time throughout hospitalization: Day I (admission date) - Day 9 (day of discharge). Patient received intravenous steroids at approximately 36 hours into hospitalization.

Although hypocoagulable states have been noted in the literature, most studies indicate an associated hypercoagulable state. There appears to be an increase in thrombinanti-thrombin complex and a decrease in antithrombin III activity, which causes an increase in thrombin generation[10,12,13]. Other studies have demonstrated an increase in fibrinogen content, increase Factor VIII, and Factor IX activity, platelet count and aggregation rate[9,12]. These hypercoagulable abnormalities return towards normal with therapy in direct correlation with sedimentation rate and clinical disease activity [12], but can still show mild abnormalities despite clinical remission[14]. 


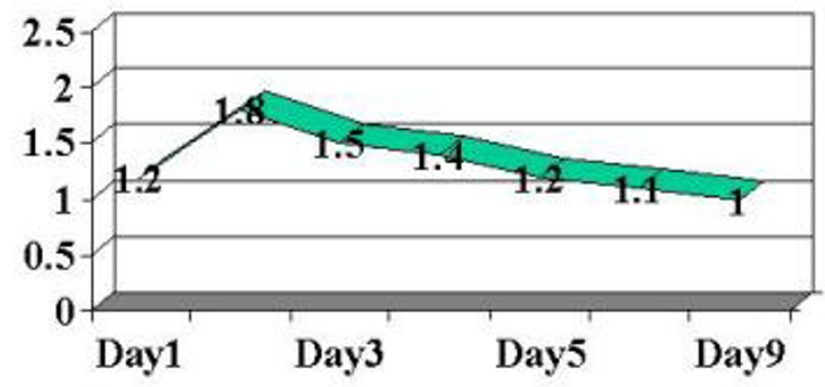

International normalized ration (INR)

Figure 6

Graphic illustration of international normalized ratio throughout hospitalization: Day I (admission date) - Day 9 (day of discharge). Patient received intravenous steroids at approximately 36 hours into hospitalization.

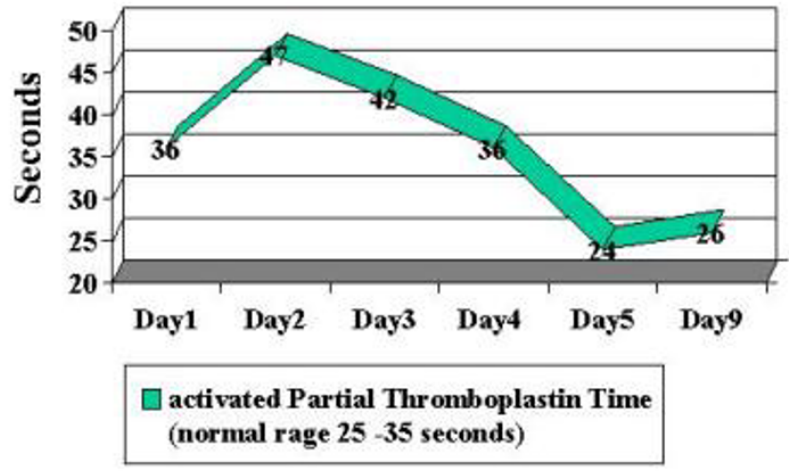

Figure 7

Graphic illustration of partial thromboplastin time C-reactive protein throughout hospitalization: Day I (admission date) Day 9 (day of discharge). Patient received intravenous steroids at approximately 36 hours into hospitalization.

The hypercoagulable state in ulcerative colitis is associated thromboembolic events; although uncommon, deep vein thrombosis, pulmonary embolisms and stroke have been associated with ulcerative colitis[6,15-18]. Disseminated intravascular coagulopathy is a rare occurrence in inflammatory bowel disease. When it occurs, it is usually associated with other co-founding problems such as septicemia, toxic megacolon or surgery.

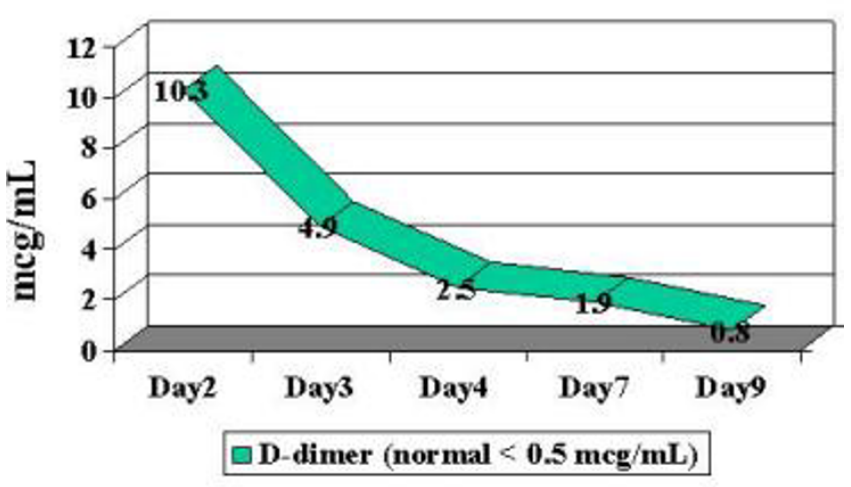

Figure 8

Graphic illustration of D-dimer throughout hospitalization: Day I (admission date) - Day 9 (day of discharge). Patient received intravenous steroids at approximately 36 hours into hospitalization.

Presented is a case of DIC associated solely with an ulcerative colitis flare resulting in a bladder hematoma. We presume that the occurrence of DIC in this patient resulted from an acute flare on top of a chronic unremitting course of ulcerative colitis. A thorough infectious work-up of this patient did not reveal any infectious etiology that would have predisposed him to develop DIC. The presumed cause of the DIC was damage to the endothelial wall of the colonic blood vessels, which exposed blood to excessive amounts of tissue factor. This in turn led to the excessive generation of thrombin and a failure of the normal coagulation inhibitory pathways. By treating the ulcerative colitis flare, we decreased the intestinal inflammation and thereby decreased the endothelial cell damage. This, theoretically, resolved the DIC. Patient's clinical symptoms and laboratory values normalized after treatment with intravenous steroids, completely resolving the disseminated intravascular coagulopathy.

\section{Competing interests}

The authors declare that they have no competing interests.

\section{Authors' contributions}

DLS drafted the manuscript. KM and DC participated in the manuscript preparation. All authors approved the final manuscript.

\section{References}

I. Bick RL: Disseminated intravascular coagulation: a review of etiology, pathophysiology, diagnosis, and management: guidelines for care. Clin Appl Thromb Hemost 2002, 8: I-3I.

2. Arai H, Hanai H, Furuta T, Sato Y, Yamada M, Kaneko E, Baba S, Sugimura $\mathrm{H}$ : A patient who survived total colonic type ulcerative colitis complicated by toxic megacolon, disseminated intravascular coagulation, methicillin-resistant Staphylococcus 
aureus infection and bilateral femoral phlebothrombosis. J Gastroenterol 1999, 34:395-399.

3. Lo D: Klebsiella septicaemia, disseminated intravascular coagulation and ulcerative colitis in an Australian Aboriginal. Med J Aust I97I, I: 1279-1280.

4. Orii S, Chiba T, Nakadate I, Fujiwara T, Ito N, Ishii M, Oana S, Chida T, Kudara N, Terui T, Yamaguchi T, Suzuki K: Pleuropericarditis and disseminated intravascular coagulation in ulcerative colitis. J Clin Gastroenterol 200 I, 32:25I-254.

5. Wong TZ, Welch JP, Holt JB: Intraoperative disseminated intravascular coagulation in a patient with ulcerative colitis. Conn Med 1 989, 53:577-578.

6. Jackson LM, O'Gorman PJ, O'Connell J, Cronin CC, Cotter KP, Shanahan F: Thrombosis in inflammatory bowel disease: clinical setting, procoagulant profile and factor $\mathbf{V}$ Leiden. Qjm 1997, 90:183-188.

7. Krasinski SD, Russell RM, Furie BC, Kruger SF, Jacques PF, Furie B: The prevalence of vitamin $\mathrm{K}$ deficiency in chronic gastrointestinal disorders. Am J Clin Nutr 1985, 41:639-643.

8. Mones RL: Thrombocytopenia and hypofibrinogenemia in association with inflammatory bowel disease. J Pediatr Gastroenterol Nutr 1983, 2:175-177.

9. Mori K, Watanabe H, Hiwatashi N, Sugai K, Goto Y: Studies on blood coagulation in ulcerative colitis and Crohn's disease. Tohoku J Exp Med 1980, 132:93-101.

10. Souto JC, Martinez E, Roca M, Mateo J, Pujol J, Gonzalez D, Fontcuberta J: Prothrombotic state and signs of endothelial lesion in plasma of patients with inflammatory bowel disease. Dig Dis Sci 1995, 40:1883-1889.

II. Weber P, Husemann S, Vielhaber H, Zimmer KP, Nowak-Gottl U: Coagulation and fibrinolysis in children, adolescents, and young adults with inflammatory bowel disease. J Pediatr Gastroenterol Nutr 1999, 28:4 18-422.

12. Lake AM, Stauffer JQ, Stuart MJ: Hemostatic alterations in inflammatory bowel disease: response to therapy. Am J Dig Dis 1978, 23:897-902.

13. Kapsoritakis AN, Potamianos SP, Sfiridaki Al, Koukourakis MI Koutroubakis IE, Roussomoustakaki MI, Manousos ON, Kouroumalis EA: Elevated thrombopoietin serum levels in patients with inflammatory bowel disease. Am J Gastroenterol 2000, 95:3478-348I.

14. van Bodegraven AA, Schoorl M, Linskens RK, Bartels PC, Tuynman $H A$ : Persistent activation of coagulation and fibrinolysis after treatment of active ulcerative colitis. Eur J Gastroenterol Hepatol 2002, 14:413-4|8.

15. Keene DL, Matzinger MA, Jacob PJ, Humphreys P: Cerebral vascular events associated with ulcerative colitis in children. Pediatr Neurol 200I, 24:238-243.

16. Solem CA, Loftus EV, Tremaine WJ, Sandborn WJ: Venous thromboembolism in inflammatory bowel disease. Am J Gastroenterol 2004, 99:97-101.

17. Sood A, Midha V, Sood N, Kaushal V: Hepatic vein thrombosis with ulcerative colitis. Indian J Gastroenterol 2000, I9: I45-I 46.

18. Bernstein CN, Blanchard JF, Houston DS, Wajda A: The incidence of deep venous thrombosis and pulmonary embolism among patients with inflammatory bowel disease: a populationbased cohort study. Thromb Haemost 200I, 85:430-434.

\section{Pre-publication history}

The pre-publication history for this paper can be accessed here:

\section{http://www.biomedcentral.com/1471-230X/4/26/pre} pub
Publish with Biomed Central and every scientist can read your work free of charge

"BioMed Central will be the most significant development for disseminating the results of biomedical research in our lifetime. "

Sir Paul Nurse, Cancer Research UK

Your research papers will be:

- available free of charge to the entire biomedical community

- peer reviewed and published immediately upon acceptance

- cited in PubMed and archived on PubMed Central

- yours - you keep the copyright

Submit your manuscript here:

http://www.biomedcentral.com/info/publishing_adv.asp
BioMedcentral 